\title{
On the Poisson brackets of differentiable generators in classical field theory
}

\author{
J. David Brown \\ Center for Theoretical Physics, The University of Texas at Austin, Austin, Texas 78712 \\ Marc Henneaux ${ }^{a}$ \\ Faculté des Sciences, Université Libre de Bruxelles, Campus Plaine C. P. 231, B-1050, Bruxelles, Belgium ${ }^{\text {b) }}$ \\ and Centro de Estudios Científicos de Santiago, Casilla 16443, Santiago 9, Chile
}

(Received 10 June 1985; accepted for publication 16 September 1985)

The canonical formulation of field theory on open spaces is considered. It is proved, under appropriate assumptions, that the Poisson bracket of two differentiable generators is also a differentiable generator.

\section{INTRODUCTION}

The following question naturally arises in the study of the Hamiltonian formulation of Einstein theory on open spacelike sections. Given the generators of two asymptotic symmetry transformations, supplemented by the appropriate surface terms at spatial infinity that make them differentiable (i.e., that make their functional derivatives well defined), ${ }^{1,2}$ can it be taken for granted ${ }^{3}$ that their Poisson bracket is also a differentiable generator? In this paper, we analyze this question and answer it affirmatively.

Because the theorem to be derived here can be applied to more general situations than just general relativity, we will adopt notations that do not explicitly refer to that case. The canonical variables will be denoted by $y^{4}(x)$ and the Poisson bracket of two differentiable functionals $F[y], G[y]$ of the canonical variables will be denoted by

$$
[F, G]=\int \frac{\delta F}{\delta y^{A}(x)} \frac{\delta G}{\delta y^{B}(x)} \sigma^{A B} d^{n} x,
$$

where $\sigma^{A B}$ is, for simplicity, a constant anstisymmetric matrix. In (1), $\delta F / \delta y^{A}(x)$ is the functional derivative of $F$ with respect to $y^{A}(x)$, defined by

$$
\delta F=\int \frac{\delta F}{\delta y^{A}(x)} \delta y^{A}(x) d^{n} x
$$

for any allowed variation $\delta y^{A}$ of the fields. The functional $F$ is said to be differentiable if its variation can indeed be brought to the form (2), with functional derivatives $\delta F$ / $\delta y^{4}(x)$ that are regular functions of $x$.

The formula (1) can be rewritten in various useful ways as follows:

$$
\begin{aligned}
{[F, G] } & =\delta_{F} G=-\delta_{G} F \\
& =\int \frac{\delta G}{\delta y^{A}(x)} \delta_{F} y^{A}(x) d^{n} x,
\end{aligned}
$$

with

$$
\delta_{F} y^{A}(x)=\frac{\delta F}{\delta y^{B}(x)} \sigma^{B A}
$$

In the open case considered here, the field configurations $y^{A}(x)$ are restricted by appropriate boundary conditions at spatial infinity, which read

\footnotetext{
a) Chercheur qualifié au Fonds National Belge de la Recherche Scientifique

b) Permanent address.
}

$$
\chi^{\alpha}\left(y^{A}, y_{i}^{A}, \ldots, y_{i_{1} \cdots i_{q}}^{A}, x^{j}\right) \rightarrow 0,
$$

for some given functions $\chi^{\alpha}$ of the fields and their derivatives

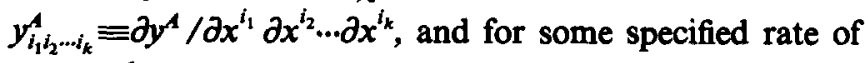
approach.

If the differentiable functional $G$ is such that $y^{A}(x)+\delta_{G} y^{A}(x)$ obeys the boundary conditions whenever $y^{A}(x)$ does, $G$ is said to be a differentiable generator. In that case the infinitesimal canonical transformation generated by $G$ maps an allowed configuration on to another allowed configuration.

From now on, $F$ and $G$ will always stand for differentiable generators which are given by integrals of local densities,

$$
\begin{aligned}
& F=\int f\left(y^{A}, y_{k}^{A}, y_{k l}^{A}, \ldots, y_{k_{1} \cdots k_{m}}^{A}, x^{i}\right) d^{n} x, \\
& G=\int g\left(y^{A}, y_{k}^{A}, y_{k l}^{A}, \ldots, y_{k_{1} \cdots k_{p}}^{A}, x^{i}\right) d^{n} x .
\end{aligned}
$$

Moreover, we assume that $f$ and $g$ are differentiable (as many times as needed) functions of their arguments. This case covers all field theoretical models of common interest.

From (6), it is easy to obtain ${ }^{4}$

$$
\delta F=\int d^{n} x \frac{\delta f}{\delta y^{A}} \delta y^{4}+\oint d^{n-1} S_{k} V_{f}^{k}(\delta y),
$$

where the following conventions have been adopted:

$$
\begin{gathered}
\frac{\delta f}{\delta y^{A}}=\frac{\partial f}{\partial y^{A}}-\partial_{k} \frac{\partial f}{\partial y_{K}^{A}}+\cdots+(-)^{m} \partial_{k_{1} \cdots k_{m}} \frac{\partial f}{\partial y_{k_{1} \cdots k_{m}}^{A}}, \\
V_{f}^{k}(\delta y)=\frac{\delta f}{\delta y_{k}^{A}} \delta y^{A}+\frac{\delta f}{\delta y_{k l}^{A}} \delta y_{l}^{A}+\cdots+\frac{\delta f}{\delta y_{k_{2} \cdots l_{m}}^{A}} \delta y_{l_{2} \cdots l_{m}}^{A}, \\
\frac{\delta f}{\delta y_{k_{1} \cdots k_{j}}^{A}}=\frac{\partial f}{\partial y_{k_{1} \cdots k_{j}}^{A}}-\partial_{k_{j+1}} \frac{\partial f}{\partial y_{k_{1} \cdots k_{j+1}}^{A}} \\
\quad+\cdots+(-)^{m-j} \partial_{k_{j+1} \cdots k_{m}} \frac{\partial f}{\partial y_{k_{1} \cdots k_{m}}^{A}} .
\end{gathered}
$$

The functions $\delta f / \delta y_{k_{1} \cdots k_{j}}^{A}$ are the "variational derivatives" of $f$ with respect to $y_{k_{1} \cdots k_{j}}^{A}$.

Since the (already improved) functional $F$ is differentiable by hypothesis, its variation (7) must reduce to the form written in (2) for any $\delta y^{A}$ that preserves the boundary conditions (5). For the case in which the variations $\delta y^{A}$ are of compact support but otherwise arbitrary, the surface inte- 
gral in (7) vanishes and the variation $\delta F$ indeed reduces to the form (2). It then follows that at each point $x$,

$$
\frac{\delta F}{\delta y^{A}(x)}=\frac{\delta f}{\delta y^{A}}
$$

where the right-hand side of $(9)$ is understood to be evaluated at $x$. Now by comparing (7) with (2) for arbitrary variations $\delta y^{A}$ compatible with the boundary conditions, the surface integral is seen to vanish,

$$
\oint d^{n-1} S_{k} V_{f}^{k}(\delta y)=0,
$$

for all allowed $\delta y^{A}$. Similar expressions hold for $G$.

We are now in a position to formulate the main theorem of our paper.

Theorem: The bracket $[F, G]$ of two differentiable generators is itself a differentiable generator.

This theorem contains two assertions. The first one is that $[F, G]$ is differentiable. The second one is that $\delta_{[F, G]} y^{A}$ has the correct asymptotic behavior, i.e., that $[F, G]$ generates a canonical transformation that preserves the boundary conditions.

\section{PROOF OF THE THEOREM}

In order to prove the theorem, we first demonstrate the following lemmas.

Lemma 1: Let $\quad \mu^{A}\left(y^{A}, y_{i}^{A}, \ldots, y_{i_{1} \cdots i_{s}}^{A}, x^{j}\right)$ and $v^{A}\left(y^{A}, y_{i}^{A}, \ldots, y_{i}^{A} \ldots i, x^{j}\right)$ be two allowed, field-dependent, "infinitesimal" variations of $y^{A}$ (i.e., let $y^{A}+\mu^{A}$ and $y^{A}+v^{A}$ obey the boundary conditions). Then, the bracket $[\mu, v]^{A}$ defined by

$$
[\mu, v]^{A}=\delta_{\mu} v^{A}-\delta_{v} \mu^{A}
$$

is also an allowed infinitesimal variation of $y^{A}$. In (11), $\delta_{\mu} v^{A}$ stands for the change in $v^{A}$ induced by the variation $\mu^{A}$,

$$
\delta_{\mu} \nu^{A}=\sum_{k=0}^{t} \frac{\partial v^{A}}{\partial y_{i_{1} \cdots i_{k}}^{c}} \mu_{i_{1} \cdots i_{k}}^{c} .
$$

The proof of this lemma is straightforward. Infinitesimal variations $\delta y^{A}$ are indeed characterized by

$$
\delta \chi^{\alpha}=\sum_{k=0}^{q} \frac{\partial \chi^{\alpha}}{\partial y_{i_{1} \cdots i_{k}}^{A}} \delta y_{i_{1} \cdots i_{k}}^{A} \rightarrow 0 .
$$

Also, note that $\delta_{\{\mu, \nu} \chi^{\alpha}$ is equal to $\delta_{\mu} \delta_{v} \chi^{\alpha}-\delta_{v} \delta_{\mu} \chi^{\alpha}$ [use $\left.\delta_{\mu} h_{i_{1} \cdots i_{k}}=\left(\delta_{\mu} h\right)_{i_{1} \ldots i_{k}}\right]$. Then since $\delta_{\nu} \chi^{\alpha}$ and $\delta_{\mu} \chi^{\alpha}$ fall off for all admissible field configurations, it follows successively that $\delta_{\mu} \delta_{v} \chi^{\alpha}, \delta_{v} \delta_{\mu} \chi^{\alpha}$, and $\delta_{[\mu, v]} \chi^{\alpha}$ also go to zero at infinity and thus, that $[\mu, v]^{A}$ preserves the boundary conditions.

Lemma 2: Let $\mu^{A}$ and $v^{A}$ be as above. In performing the integrations by parts needed to transform

$$
\int \delta_{\nu}\left(\frac{\delta f}{\delta y^{B}}\right) \cdot \mu^{B} d^{n} x
$$

into a volume piece containing only undifferentiated $v^{A}$ plus a surface integral, that surface integral is simply given by

$$
\oint d^{n-1} S_{k} A_{f}^{k}(\mu, v),
$$

$$
A_{f}^{k}(\mu, v)=\delta_{\mu} V_{f}^{k}(v)-\delta_{v} V_{f}^{k}(\mu)+V_{f}^{k}([v, \mu]) .
$$

This can be seen by using again the simple identity

$$
\delta_{\nu} \delta_{\mu} f-\delta_{\mu} \delta_{v} f=\delta_{[v, \mu]} f \text {. }
$$

By integrating (15) over $x$ and taking into account (7) and the definition of the bracket $[v, \mu]^{A}$, this yields

$$
\begin{aligned}
\int \delta_{v}\left(\frac{\delta f}{\delta y^{A}}\right) \mu^{A} d^{n} x= & \int \delta_{\mu}\left(\frac{\delta f}{\delta y^{4}}\right) v^{A} d^{n} x \\
& +\oint d^{n-1} S_{k} A_{f}^{k}(\mu, v),
\end{aligned}
$$

where $A_{f}^{k}(\mu, v)$ is given by (14). Since the volume integral on the right-hand side of (16) contains no derivative of $v^{A}$, the second lemma is proved.

Incidentally, (16) shows that the polynomials $\delta_{v}(\delta f)$ $\delta y^{4}$ ) are "self-adjoint," as indeed they should be (see, e.g., Ref. 4, pp. 202-204).

Lemma 3: Let $\mu^{A}$ and $v^{A}$ be given by

$$
\mu^{A}=\delta_{F} y^{A}=\frac{\delta f}{\delta y^{B}} \sigma^{B A}, \quad v^{A}=\delta_{G} y^{A}=\frac{\delta g}{\delta y^{B}} \sigma^{B A} .
$$

The bracket $[\mu, v]^{A}$ is then equal to

$$
[\mu, v]^{A}=\frac{\delta}{\delta y^{B}}\left(\frac{\delta f}{\delta y^{C}} \frac{\delta g}{\delta y^{D}} \sigma^{C D}\right) \sigma^{B A} .
$$

This is a straightforward consequence of the following identity of the calculus of variations:

$$
\frac{\delta}{\delta y^{A}}\left(\frac{\delta f}{\delta y^{B}} \frac{\delta g}{\delta y^{C}} \sigma^{B C}\right)=\delta_{F} \frac{\delta g}{\delta y^{A}}-\delta_{G} \frac{\delta f}{\delta y^{A}}
$$

(where $\delta_{F}=\delta_{\mu}, \delta_{G}=\delta_{v}$ ).

To prove this identity, integrate the right-hand side of (18), multiplied by an arbitrary function $\epsilon^{A}$ of compact support, over the whole space and make all necessary integrations by parts. The resulting surface integrals will all vanish since $\epsilon^{A}$ is identically zero sufficiently close to spatial infinity. This gives, using the equality (16),

$$
\begin{aligned}
\int \epsilon^{A} & \left(\delta_{F} \frac{\delta g}{\delta y^{A}}-\delta_{G} \frac{\delta f}{\delta y^{A}}\right) d^{n} x \\
& =\int\left(\delta_{F} y^{A} \delta_{\epsilon} \frac{\delta g}{\delta y^{A}}-\delta_{G} y^{A} \delta_{\epsilon} \frac{\delta f}{\delta y^{A}}\right) d^{n} x \\
& =\int \delta_{\epsilon}\left(\frac{\delta f}{\delta y^{A}} \frac{\delta g}{\delta y^{B}} \sigma^{A B}\right) d^{n} x .
\end{aligned}
$$

Again making an integration by parts and taking the arbitrariness of $\epsilon^{A}$ into account, (18) is easily seen to hold true.

We now turn to the demonstration of the theorem itself. The first step in the demonstration amounts to showing that $[F, G]$ is differentiable. This is done by relating the surface term that appears in the variation of $[F, G]$ to the surface terms that arise in the variations of $F$ and $G$, and that are known to vanish.

The definition (1) and the equality (9) may be used to write the variation of $[F, G]$ for an arbitrary (allowed) variation $v^{A}$ of $y^{A}$ as

$\delta_{v}[F, G]=\int \delta_{v}\left(\frac{\delta g}{\delta y^{B}}\right) \delta_{F} y^{B} d^{n} x-\int \delta_{v}\left(\frac{\delta f}{\delta y^{B}}\right) \delta_{G} y^{B} d^{n} x$

with 
We want to prove that the surface integrals arising from the appropriate integrations by parts in each term of the right-hand side of $(20)$ are separately zero. Since these terms have the same structure, it is enough to show this property for one of them.

From the second lemma, we know that the surface integral arising in $\int \delta_{v}\left(\delta f / \delta y^{B}\right) \delta_{G} y^{B} d^{n} x$ is given by $\oint A_{f}^{k}\left(\delta_{G} y, v\right) d^{n-1} S_{k}$ [formula (14)], where

$$
A_{f}^{k}\left(\delta_{G} y, v\right)=\delta_{G} V_{f}^{k}(v)-\delta_{v} V_{f}^{k}\left(\delta_{G} y\right)+V_{f}^{k}\left(\left[v, \delta_{G} y\right]\right) .
$$

But each of the terms in $A_{f}^{k}\left(\delta_{G} y, v\right)$ contribute zero, because (i) $\oint V_{f}^{k}(v) d^{n-1} S_{k}$ vanishes for any allowed variation performed about any allowed configuration, and so also does

$$
\oint \delta_{G} V_{f}^{k}(v) d^{n-1} S_{k}=\delta_{G} \oint V_{f}^{k}(v) d^{n-1} S_{k} ;
$$

(ii) similary, $\oint \delta_{v} V_{f}^{k}\left(\delta_{G} y\right) d^{n-1} S_{k}$ vanishes for an identical reason; and (iii) $\left[v, \delta_{G} y\right]^{A}$ is an allowed variation by virtue of the first lemma, and so its flux integral $\oint V_{f}^{k}\left(\left[v, \delta_{G} y\right]\right) d^{n-1} S_{k}$ is also zero. Hence, there is no unwanted surface term in $\delta_{\nu}[F, G]$.

This proves that the Poisson bracket $[F, G]$ is differentiable; its functional derivatives are equal to [see (1) and (9)],

$$
\frac{\delta[F, G]}{\delta y^{A}(x)}=\frac{\delta}{\delta y^{A}}\left(\frac{\delta f}{\delta y^{B}} \frac{\delta g}{\delta y^{C}}\right) \sigma^{B C} .
$$

Then the result of the third lemma can be rewritten as

$$
\delta_{[F, G]} y^{A}=\left[\delta_{F,} \delta_{G}\right] y^{A},
$$

and it follows from the first lemma that $\delta_{[F, G]} y^{A}$ is an allowed variation. This shows that the canonical transformation generated by $[F, G]$ preserves the boundary conditions and completes the demonstration of the theorem.

The conclusion of our paper is that as soon as the canonical generators of a field theory are supplemented, in the spatially open case, by the appropriate surface terms needed to make them well defined, then their Poisson brackets may be taken as many times as desired (provided these generators are given by the integrals of $C^{\infty}$ local densities). In that sense, the problem of the surface terms arises only once. In addition, the Poisson brackets possess the nice properties expected from classical mechanics with a finite number of degrees of freedom [cf. (23)].

As a final point, we note that the results of our paper can be easily extended to the case when some of the variables $y^{A}(x)$ are fermionic.

\section{ACKNOWLEDGMENTS}

It is a pleasure to thank Jules Géhéniau and Claudio Teitelboim for useful discussions.

This work has been supported in part by the National Science Foundation under Grant No. PHY-8216715 and by research funds from the University of Texas at Austin, Center for Theoretical Physics.

${ }^{1}$ The need to supplement the Dirac-A.D.M. Hamiltonian of general relativity by appropriate surface terms at spatial infinity has been pointed out, in the asymptotically flat case, by Regge and Teitelboim [T. Regge and C. Teitelboim, Ann. Phys. N.Y. 88, 286 (1974)]. As shown by the authors, the surface terms yield the A.D.M. energy-momentum. These ideas have been further discussed in Y. Choquet-Bruhat, A. Fischer, and J. Marsden, "Maximal hypersurfaces and positivity of mass," in Proceedings of the Enrico Fermi Summer School of the Italian Physical Society, Course LXVII, edited by J. Ehlers (North-Holland, Amsterdam, 1979).

${ }^{2}$ The extension of the results of Ref. 1 to the asymptotically anti-de Sitter case has been done in M. Henneaux and C. Teitelboim, Phys. Lett. B 142, 355 (1984); M. Henneaux and C. Teitelboim, Commun. Math. Phys. 98, 391 (1985).

${ }^{3}$ This question is important for deriving the Poisson bracket algebra of the canonical generators of asymptotic symmetries, and has been particularly emphasized in J. D. Brown and M. Henneaux, "Central charges in the canonical realization of asymptotic symmetries: An example from threedimensional gravity," to be published in Commun. Math. Phys. See also A. Ashtekar, "Asymptotic properties of isolated systems: Recent developments," in General Relativity and Gravitation, edited by B. Bertotti, F. de Felice, and A. Pascolini (Reidel, Dordrecht, 1984).

"Th. De Donder, "Théorie Invariantive du Calcul des Variations (Gauthier-Villars, Paris, 1935), 2nd. ed. 